\title{
Promoting Clinical Practices to Sleep Better: A Dream Intensive Care Unit?
}

\author{
Nagarajan Ramakrishnan
}

Indian Journal of Critical Care Medicine (2020): 10.5005/jp-journals-10071-23338

Sleep abnormalities are frequently noted in intensive care unit (ICU) patients. ${ }^{1}$ These include sleep deprivation and fragmented sleep pattern with abnormal sleep architecture. Day-night disorientation and sleeping during daytime hours are often noted with a shift toward lighter stages of sleep at night. Poor sleep pattern may contribute to brain dysfunction, of which delirium is a manifestation. Sedation is indeed a poor substitute for sleep, and it would be best to promote nonpharmacological clinical practices. Ventilator modes, music, reduction of ambient noise, use of ear plugs, appropriate lighting to promote day-night rhythm, and scheduling patient care activities appropriately to reduce interventions at night have all been considered. Unfortunately, it is still noted that pharmacological measures are used more often in most ICUs. Although sleep is often not an area of focus in critical care, it is heartening to note two publications in the same issue of Indian Journal of Critical Care Medicine (IJCCM) from different centers addressing the impact of light and sound on sleep in ICU.

The single night observational study by Czempik et al. ${ }^{2}$ on impact of light may have limitations but still "enlightens" the readers on this issue. The authors have neither excluded those with prior sleep problems nor recorded the impact of other factors, which is a significant limitation. Moreover, using a sleep observation tool is an inferior method of assessing sleep quality. Although polysomnography (PSG) with multiparameter testing is still the gold standard, it remains impractical to be performed on a regular basis raising the question of alternative tools that may be easier to use. Bispectral index is used for assessing the depth of sedation but its use for sleep assessment is poorly documented. Actigraphy although simpler to use has been shown to consistently overestimate total sleep time and efficiency and underestimate nocturnal awakenings, which are more frequently reported by nurse assessment tools and patient questionnaires. ${ }^{3}$ Despite its limitations, the authors could have considered using Actigraph as an objective tool as it also has light sensors that could have been used as a uniform tool for light measurement. In their efforts to have a "practical study" without any additional objective measures of sleep, the authors have possibly missed the opportunity for a more robust statement on whether the "lights off" practices are essential or should we just limit the intensity of light. More importantly, options such as actigraphy could have helped identify daytime sleep and total sleep time during the 24-hour period. This would be important as daytime sleep would likely occur when patients are exposed to higher intensity of light. Current international ICU guidelines recommend natural daylight for every patient room and artificial light that can be dialed up and down; currently, there is no indication as to what daytime light intensity should be achieved. ${ }^{4}$ The guidelines by Indian Society of Critical Care Medicine (ISCCM) on ICU planning and design makes recommendations for lighting in the
Department of Critical Care Medicine, Apollo Hospitals, Chennai, Tamil Nadu, India

Corresponding Author: Nagarajan Ramakrishnan, Department of Critical Care Medicine, Apollo Hospitals, Chennai, Tamil Nadu, India, Phone: +91 44 28296517, e-mail: icudoctor@gmail.com

How to cite this article: Ramakrishnan N. Promoting Clinical Practices to Sleep Better: A Dream Intensive Care Unit? Indian J Crit Care Med 2020;24(1):1-2.

Source of support: Nil

Conflict of interest: None

room, for procedures and general patient care but not specifically for sleep promotion. ${ }^{5}$

The study on use of ear plugs and eye shades by Arttawejkul et al. ${ }^{6}$ is limited by number of patients, but is notable for efforts to mimic habitual home bedtime hours and combining subjective and objective measures. In particular, the use of the gold standard PSG and comparing it with the easier to use Actigraphy adds value. There were insignificant differences between the intervention and control groups due to several limitations listed by the authors. It is notable that the TISS-28 score was higher in the intervention group, which makes one wonder if the higher interventions at night could have been a contributory factor.

There is increasing focus on noncommunicable diseases in chronic health and wellness. Although awareness is being created for early detection and management of hypertension, diabetes, cardiovascular diseases, obesity, and cancer, it is noted that sleep disorders, which are more common and could be contributing to all of these problems, are often ignored. Similarly, in the critical care setting, hemodynamics and organ dysfunction are predominantly focused, but it is important to remember simple clinical practices that could promote circadian rhythm and sleep may impact these.

Critical illness is a time of vulnerability, and it would be important to explore all options to promote recovery. Strategies to promote consolidated, restorative sleep and maintain the circadian rhythm as best as possible could contribute to improved biological function. ${ }^{7}$ But how often do we do these? Hofhuis et al. evaluated sleep promotion practices in European $\mathrm{ICUs}^{8}$ through a selfadministered survey. It was noted that only $9 \%$ of the participating ICUs had a protocol for sleep and notably $1 \%$ of the ICUs utilized a questionnaire to assess sleep. The most often used practice to check if the patient was asleep was the nurses noticing that the patient was lying quietly with closed eyes. Variations in blood pressure and respiratory rate were other parameters considered to indicate sleep. Most common interventions were reducing ICU 
staff noise, light, and nurse interventions at night. It is time to wake up and call for action to create protocols for sleep-promoting practices in our ICUs.

\section{References}

1. Parthasarathy $\mathrm{S}$, Tobin MJ. Sleep in the intensive care unit. Intensive Care Med 2004;30(2):197-206. DOI: 10.1007/s00134-0032030-6.

2. Czempik PF, Jarosińska A, Machlowska K, Pluta M. Impact of light intensity on sleep of patients in the intensive care unit: a prospective observational study. Indian J Crit Care Med 2020;24(1):33-37.

3. Schwab KE, Ronish B, Needham DM, To AQ, Martin JL, Kamdar BB. Actigraphy to evaluate sleep in the intensive care unit: a systematic review. Ann Am Thorac Soc 2018;15(9):1075-1082. DOI: 10.1513/ AnnalsATS.201801-004OC.
4. Thompson DR, Hamilton DK, Cadenhead CD, Swoboda SM, Schwindel SM, Anderson DC, et al. Guidelines for intensive care unit design. Crit Care Med 2012;40(5):1586-1600. DOI: 10.1097/ CCM.0b013e3182413bb2.

5. Rungta N, ICU Planning and Designing in India - Guidelines 2010 Guidelines Committee ISCCM [Internet]. [cited 2020 Jan 14]. Available from: https://isccm.org/pdf/Section1.pdf.

6. Arttawejkul P, Reutrakul S, Muntham D, Chirakalwasan N. Effect of nighttime earplugs and eye masks on sleep quality in intensive care unit patients. Indian J Crit Care Med 2020;24(1):6-10.

7. Pisani MA, Friese RS, Gehlbach BK, Schwab RJ, Weinhouse GL, Jones SF. Sleep in the intensive care unit. Am J Respir Crit Care Med 2015;191(7):731-738. DOI: 10.1164/rccm.201411-2099Cl.

8. Hofhuis JGM, Rose L, Blackwood B, Akerman E, McGaughey J, Egerod I, et al. Clinical practices to promote sleep in the ICU: a multinational survey. Int J Nurs Stud 2018;81:107-114. DOI: 10.1016/ j.ijnurstu.2018.03.001. 